\title{
Intellectual disability and other neuropsychiatric outcomes in high-risk children of mothers with schizophrenia, bipolar disorder and unipolar major depression ${ }^{\dagger}$
}

Vera A. Morgan, Maxine L. Croft, Giulietta M. Valuri, Stephen R. Zubrick, Carol Bower, Thomas F. McNeil and Assen V. Jablensky

\section{Background}

Recent evidence points to partially shared genetics of neuropsychiatric disorders.

\section{Aims}

We examined risk of intellectual disability and other neuropsychiatric outcomes in 3174 children of mothers with schizophrenia, bipolar disorder or unipolar major depression compared with 3129 children of unaffected mothers.

\section{Method}

We used record linkage across Western Australian population-based registers. The contribution of obstetric factors to risk of intellectual disability was assessed.

\section{Results}

Children were at significantly increased risk of intellectual disability with odds ratios (ORs) of $3.2(95 \% \mathrm{Cl} 1.8-5.7), 3.1$ (95\% Cl 1.9-4.9) and 2.9 (95\% Cl 1.8-4.7) in the maternal schizophrenia, bipolar disorder and unipolar depression groups respectively. Multivariate analysis suggests familial and obstetric factors may contribute independently to the risk. Although summated labour/delivery complications $(\mathrm{OR}=1.4,95 \% \mathrm{Cl} 1.0-2.0)$ just failed to reach significance, neonatal encephalopathy $(\mathrm{OR}=7.7,95 \% \mathrm{Cl} 3.0-20.2)$ and fetal distress $(\mathrm{OR}=1.8,95 \% \mathrm{Cl} 1.1-2.7)$ were independent significant predictors. Rates of rare syndromes in children of mothers with mental disorder were well above population rates. Risk of pervasive developmental disorders, including autism, was significantly elevated for children of mothers with bipolar disorder. Risk of epilepsy was doubled for children of mothers with unipolar depression.

\section{Conclusions}

Our findings provide epidemiological support for clustering of neuropsychiatric disorders. Further larger epidemiological studies are warranted.

\section{Declaration of interest}

None.
The recent accumulation of data on the partially shared genetics of neuropsychiatric disorders, including schizophrenia, intellectual disability, autism and epilepsy, ${ }^{1,2}$ includes evidence of the occurrence of both inherited and de novo rare mutations (copy number variations) at multiple genomic loci especially in schizophrenia and autism. ${ }^{1,3-5}$ These findings are underpinned by observational and epidemiological data supporting a relationship between idiopathic intellectual disability and psychosis. Kraepelin estimated that intellectual disability was the basis of some $3.5 \%$ of cases of dementia praecox. ${ }^{6}$ In 1938, Penrose reported that $3.8 \%$ of a carefully ascertained and representative sample of 1280 patients with intellectual disability in Colchester had schizophrenia and $1.9 \%$ had affective psychoses. ${ }^{7}$ Commonly reported estimates still put the risk of schizophrenia in intellectually disabled populations at around $3 \%{ }^{8}$ Our own estimate, based on whole-of-population recordlinked data, is $4.9 \%$ ( $4.5-5.2 \%$ depending on birth cohort) for the prevalence of intellectual disability in schizophrenia and $4.4 \%$ (3.7-5.2\% depending on birth cohort) for the prevalence of schizophrenia in intellectual disability. ${ }^{9}$ This compares with a lifetime morbid risk in the general population of around 1\% both for intellectual disability ${ }^{10}$ and for schizophrenia. ${ }^{11}$ A growing number of studies show substantial familiality across the two disorders. In particular, there are reports of increased rates of schizophrenia in families of probands with intellectual disability; intellectual disability in families of probands with schizophrenia;

†See editorial, pp. 268-269, this issue. and multiply affected families with co-occurring intellectual disability and schizophrenia (see online Tables DS1-DS3).

There have been few population-based epidemiological studies of clustering of neuropsychiatric disorders. The longitudinal cohort of high-risk children that we have identified using whole-of-population administrative and clinical registers offers a unique opportunity to examine the extent to which schizophrenia, intellectual disability and other neuropsychiatric disorders share a common aetiological basis. Consequently, the aims of this study are: (a) to determine the risk of intellectual disability, rare syndromes, pervasive developmental disorders, convulsions and epilepsy in a population-based cohort of children of women with schizophrenia compared with children of women with no recorded psychiatric history; (b) to examine the role of obstetric complications in mediating the risk of intellectual disability; and (c) to assess the specificity of findings to maternal schizophrenia compared with maternal bipolar disorder and unipolar major depression.

\section{Method}

\section{Participants}

This study is part of a larger programme of work using whole-ofpopulation health registers in Western Australia to disentangle environmental and familial contributions to risk of adverse neuropsychiatric outcomes in a cohort of children at familial high risk of psychosis having been born to women with a lifetime diagnosis of a psychotic illness. To create the cohort, the Western 
Australian midwives' database (308 022 births) was linked to the psychiatric case register, which includes in- and out-patient contacts since 1966 (79599 women), to identify children born in 1980-1992 to mothers with schizophrenia (382 mothers, 618 children), bipolar disorder (763 mothers, 1301 children) and unipolar major depression (686 mothers, 1255 children). An unmatched group of unaffected mothers was randomly selected from all mothers on the midwives' database who had given birth in 1980-1992 and who had no record of psychiatric illness. Their children formed the comparison group (1831 mothers, 3129 children). This cohort and the underlying system of linkages across health and other whole-of-population databases are described in detail elsewhere. ${ }^{12,13}$ Maternally linked sibships among children born in 1980-1992 were established using the midwives' birth records and the fathers of index children were identified using linkage to registration records. ${ }^{14,15}$

\section{Classification of psychiatric status and intellectual disability}

Data on the psychiatric status of mothers, fathers and children came from the psychiatric case register which uses the ICD to record diagnosis. Codes from ICD- $9^{16}$ used to identify specific disorders were: schizophrenia (295.0-295.9), bipolar disorder (296.0, 296.2-296.5) and unipolar major depression (296.1, 296.6, 296.8, 296.9). The concurrent validity of register diagnoses of schizophrenia and affective psychoses have been evaluated against an independent sample assessed using a semi-structured diagnostic interview ${ }^{17}$ with sensitivity of 0.92 and specificity of 0.88 for schizophrenia and 0.80 and 0.90 respectively for affective psychoses. ${ }^{12}$

Cognitive status was determined using intellectual disability data from three sources. The main source was the Western Australian intellectual disability register. It is estimated to cover over $75 \%$ of Western Australian cases, with under-ascertainment most likely to affect borderline cases. Registration is required for receipt of services, so coverage increases with age. American Association for Mental Deficiency criteria are used to define intellectual disability, namely an IQ that is two or more standard deviations below the population mean and specific limitations in adaptive behaviours and skills. The register has additional fields, including Heber diagnoses, ${ }^{18}$ that indicate the aetiological basis of the disability. As the Heber system is quite old, one of the authors (V.M.), in conjunction with an expert in the field, reorganised Heber codes into hierarchical categories that were more consistent with current classification systems to indicate a likely aetiological basis of biomedical origin (genetic, metabolic or teratogenic) of the intellectual disability. This approach, based broadly on categories developed by Yeargin-Allsopp, is described elsewhere. ${ }^{9,19}$ As not all school-aged children are in receipt of disability services, intellectual disability register data were supplemented with Western Australian education department learning disability data that use the IQ criterion to identify intellectual disability. Finally, the psychiatric case register was checked to see if there were any children not already identified who had a diagnosis of intellectually disability (ICD-9 317-319). Where multiple data sources existed for the one person, a hierarchy was applied with preference given to intellectual disability services data, followed by education department data, and then data on the psychiatric case register. For this study, people with borderline low IQ (scores of 70-74) were included.

Rare syndromes were identified using the statewide registers for birth defects and intellectual disability and included Hurler, Klinefelter, Moebius, Noonan, Prader-Willi, Rett, RubinsteinTaybi, VATER (vertebrae, anus, trachea, oesophagus, renal) association and Turner syndromes. These are predominantly cognitively impairing rare syndromes. Pervasive developmental disorders were identified using the intellectual disability register. Therefore we report only on cases of pervasive developmental disorder with co-occurring intellectual disability.

\section{Obstetric complications}

Data on obstetric complications were taken from the midwives' database, which includes mandatory, prospectively collected data on all infants born in Western Australia at 20 weeks gestation or greater or weighing at least $400 \mathrm{~g}$, including home births, and not restricted to live births. Core data included prospectively recorded data on maternal age, marital status and ethnicity, baby's gestational age and weight, pregnancy complications (e.g. pre-eclampsia, placenta praevia, abruption, substance misuse), labour and delivery complications (e.g. cephalopelvic disproportion, prolapsed cord, fetal distress) and early neonatal complications (e.g. intubation, 5-minute Apgar score). A proxy for neonatal encephalopathy was constructed based on clinical criteria developed by Badawi and colleagues. ${ }^{20}$ In addition, the McNeil-Sjöström Scale for Obstetric Complications was employed in the study. This scale was selected because it is underpinned by strong biological considerations, including timing of the complication and likely impact on central nervous system development. Binary indicators of complications of the pregnancy and labour/delivery period were created using the scale. Previous research, including our own, ${ }^{12}$ has shown that binary scores based on cut-offs for severity ratings of the specific obstetric complications provide an effective tool for identifying differences between patient and comparison groups and relationships between obstetric complications and other important characteristics. We used complications at severity level 4 or higher defined by McNeil-Sjöström as indicating severity sufficient to be potentially clearly harmful or relevant to central nervous system development. ${ }^{21}$ The algorithm underlying this scale has been validated. ${ }^{12}$

\section{Convulsions}

Convulsions (ICD-9 779.0, 780.3x-780.39; ICD-1022 P90.x, R56.xx-R56.8x) and epilepsy (ICD-9 345.xx-345.99; ICD-10 G40.xx-G41.9x) were identified using the hospital morbidity register, which covers all public and private hospital admissions state-wide. Although coverage of the hospital morbidity register is comprehensive, all cases reported here are individuals that have been admitted to hospital and therefore represent the more serious end of the spectrum.

\section{Statistical analysis}

Frequency distributions and odds ratios (ORs) and their confidence intervals (CIs) were computed using SPSS (version 14.0.0) for Windows. Multivariate models for the risk of intellectual disability were built and fitted using the logistic regression procedure in Stata (version 9.00) for Windows which permits the adjustment of standard errors to take into account correlations within families. A series of steps was undertaken to determine the most parsimonious model best fitting the data, bearing in mind theoretical considerations. Univariate analyses were run, with each individual predictor regressed on offspring intellectual disability. The predictor variables were: parental intellectual disability (yes/no), maternal psychosis status (schizophrenia/bipolar disorder/unipolar major depression/comparison), paternal psychiatric illness (yes/no), any pregnancy complications (yes/no) and any labour/delivery complications (yes/no). Familial variables (parental intellectual disability, maternal psychosis status, paternal psychiatric illness) were then included in a single 
model and interactions were examined. In order to assess whether familial effects were mediated by obstetric complications, the model was rerun with exposure to pregnancy complications and exposure to labour/delivery complications included. Interactions were again assessed. In the most parsimonious model, familial variables and exposure to labour/delivery complications were retained. The model was then adjusted for gender of offspring, indigenous status and birth order within the family, as well as for intragroup correlations within families. To observe the impact of specific obstetric events on the outcome, the model was rerun using backward stepwise regression, with the binary labour/delivery complications variable replaced by neonatal encephalopathy as well as nine individual complications of the labour/delivery period selected a priori on the basis of previous findings of reproductive pathology in women with psychosis: ${ }^{12}$ fetal distress; postpartum haemorrhage; abruption of the placenta; cord complications; abnormal presentation; cephalopelvic disproportion; precipitate delivery; prolonged labour; and use of a narcotic antagonist. This model was adjusted as before.

\section{Results}

\section{Intellectual disability}

There were 129 cases of intellectual disability among the 6303 study children: 69 identified through the disability register, 58 on the school learning disability database and two on the psychiatric case register. Rates of intellectual disability were significantly elevated in all three groups of case children compared with comparison children. The percentages of affected offspring by maternal psychiatric status were: schizophrenia 3.2\%, bipolar disorder $3.1 \%$ and unipolar major depression $2.9 \%$, compared with $1.0 \%$ for the comparison group. The unadjusted odds ratios and their 95\% confidence intervals are given in Table 1.

Overall, gender was similarly distributed across all groups of children in the study database. However, among offspring with intellectual disability, the percentage of males was $55.0 \%$ in the maternal schizophrenia group, $65.0 \%$ in the maternal bipolar disorder group, $64.9 \%$ in the maternal unipolar depression group and $71.9 \%$ in the comparison group (Table 2). Parental intellectual disability was differentially distributed: $25.0 \%$ of affected children in the maternal schizophrenia group had at least one parent with intellectual disability, compared with $7.5 \%$ in the maternal bipolar disorder group and $9.4 \%$ in the comparison group; no affected children in the maternal unipolar depression group had a parent with intellectual disability. In addition, $20.0 \%$ of affected children in the maternal schizophrenia group had a father with a psychiatric illness recorded on the psychiatric case register, compared with $40.0 \%$ in the maternal bipolar disorder group, $32.4 \%$ in the maternal unipolar depression group and $18.7 \%$ in the comparison group. All groups of affected case children had increased rates of both pregnancy and labour/delivery complications compared with comparison children (Table 2).

Analyses were undertaken to assess the relative contribution of key variables to the risk of intellectual disability in this cohort of high-risk children. In the univariate analysis, parental intellectual disability status, maternal severe mental illness, paternal psychiatric status and exposure to labour/delivery but not pregnancy complications were significantly associated with the risk of intellectual disability (Table 3 ). Multivariate models were constructed to determine the most parsimonious model that best fitted the data (see Method). Only the main effects were significant, with no significant interactions (Table 3).

In a final analysis, the binary obstetric exposure variable was replaced by nine individual labour/delivery complications and

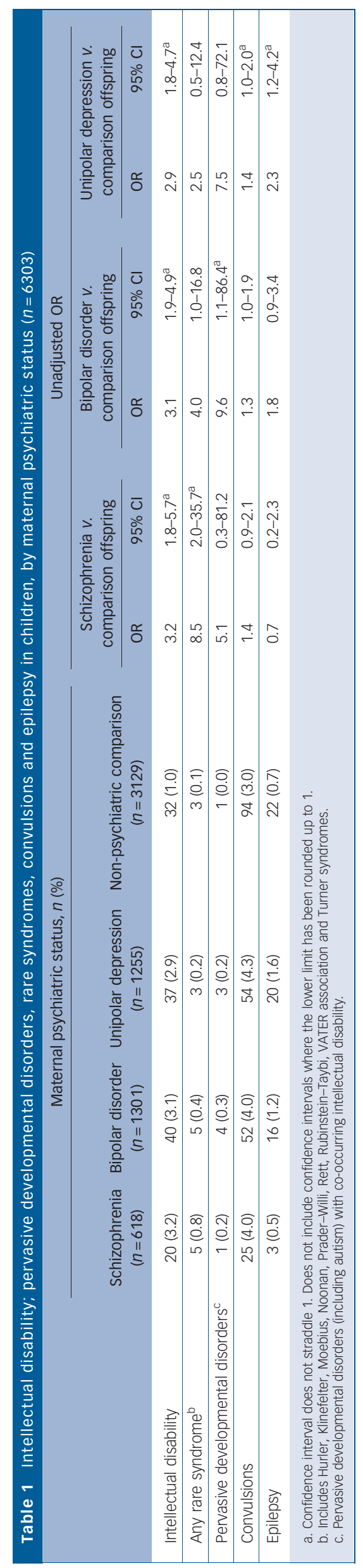




\begin{tabular}{|c|c|c|c|c|c|c|c|c|}
\hline & \multicolumn{8}{|c|}{ Maternal psychiatric status } \\
\hline & \multicolumn{2}{|c|}{ Schizophrenia $(n=20)$} & \multicolumn{2}{|c|}{ Bipolar disorder $(n=40)$} & \multicolumn{2}{|c|}{ Unipolar depression $(n=37)$} & \multicolumn{2}{|c|}{ Non-psychiatric comparison $(n=32)$} \\
\hline & $n$ & $\%$ & $n$ & $\%$ & $n$ & $\%$ & $n$ & $\%$ \\
\hline \multicolumn{9}{|l|}{ Sociodemographic } \\
\hline Male & 11 & 55.0 & 26 & 65.0 & 24 & 64.9 & 23 & 71.9 \\
\hline Indigenous & 1 & 5.0 & 3 & 7.5 & 6 & 16.2 & 4 & 12.5 \\
\hline Parental intellectual disability & 5 & 25.0 & 3 & 7.5 & 0 & 0.0 & 3 & 9.4 \\
\hline Paternal psychiatric history & 4 & 20.0 & 16 & 40.0 & 12 & 32.4 & 6 & 18.7 \\
\hline \multicolumn{9}{|l|}{ Obstetric history } \\
\hline Pregnancy complications & 7 & 35.0 & 15 & 37.5 & 12 & 32.4 & 6 & 18.8 \\
\hline Labour/delivery complications & 13 & 65.0 & 25 & 62.5 & 20 & 54.1 & 16 & 50.0 \\
\hline Neonatal encephalopathy & 1 & 5.0 & 0 & 0.0 & 3 & 8.1 & 2 & 6.3 \\
\hline
\end{tabular}

neonatal encephalopathy (see Method). Parental intellectual disability status, maternal severe mental illness and paternal psychiatric status remained significant. Neonatal encephalopathy $(\mathrm{OR}=7.7,95 \%$ CI 3.0-20.2) and fetal distress $(\mathrm{OR}=1.8,95 \%$ CI 1.1-2.7) were the only additional significant predictors.

It has been postulated that the risk of schizophrenia ${ }^{23}$ and autism $^{24}$ increases with increasing paternal age. When the analysis in Table 3 was repeated including maternal and paternal age, no association was found between maternal age or paternal age and intellectual disability in offspring. The relationship between the timing of maternal psychosis onset and the date of birth of the offspring with intellectual disability was also examined. The risk of intellectual disability in offspring was not related to the timing of the onset of maternal psychiatric illness relative to the index birth for the children of mothers with schizophrenia or unipolar major depression. However, for children of mothers with bipolar disorder, the risk of intellectual disability was significantly increased only if onset of maternal illness pre-dated the index birth.

\section{Aetiological basis of intellectual disability}

Data were available on the aetiology of the intellectual disability using the Heber classification for the 69 children on the intellectual disability register. These data were used to determine whether there was a likely aetiological basis of biomedical origin (genetic, metabolic or teratogenic) for the intellectual disability. Across the sample, the rate of intellectual disability of likely biomedical basis was higher in offspring of case mothers than offspring of comparison mothers, at 48.5 cases per 10000 population for maternal schizophrenia, 69.2 for maternal bipolar disorder and 55.8 for maternal unipolar major depression compared with 19.2 for comparison children (Table 4).

\section{Rare syndromes}

The distribution of rare syndromes among offspring was examined. Syndromes included Hurler, Klinefelter, Moebius, Noonan, Prader-Willi, Rett, Rubinstein-Taybi, VATER association and Turner syndromes. There were unusually high rates of these syndromes, differentially distributed, among the case children. These rates were well in excess of population rates (Table 5). The odds of having any one of the rare syndromes were calculated (Table 1). The risk was elevated for all three groups of case children, with the greatest risk in children of mothers with schizophrenia ( $\mathrm{OR}=8.5,95 \%$ CI 2.0-35.7). Wide confidence intervals are the result of the small numbers, affecting the precision of the estimates obtained.

\section{Pervasive developmental disorders including autism}

For the most part, it was not possible to examine individual disorders or syndromes owing to small numbers. However, there were nine children with pervasive developmental disorders and co-occurring intellectual disability so an odds ratio was calculated for this outcome (Table 1). The risk of a pervasive developmental disorder was increased significantly in the maternal bipolar disorder group.

\section{Epilepsy, convulsions}

There were 61 children who had been admitted to hospital with a diagnosis of epilepsy and 225 who had been admitted for convulsions (Table 1). Compared with the children of unaffected mothers, odds of epilepsy were increased approximately twofold in the maternal bipolar disorder and unipolar depression groups, significantly so in the latter group. The odds of being admitted to

\begin{tabular}{|c|c|c|c|c|}
\hline & \multicolumn{2}{|c|}{ Separate univariate models } & \multicolumn{2}{|c|}{ Adjusted multivariate model ${ }^{a}$} \\
\hline & Odds ratio & $95 \% \mathrm{Cl}$ & Odds ratio & $95 \% \mathrm{Cl}$ \\
\hline Parental intellectual disability & 19.1 & $9.3-39.0^{b}$ & 15.2 & $6.0-38.2^{b}$ \\
\hline \multicolumn{5}{|l|}{ Maternal severe mental illness } \\
\hline Schizophrenia & 3.2 & $1.8-5.7^{b}$ & 2.2 & $1.2-4.3^{b}$ \\
\hline Bipolar disorder & 3.1 & $1.9-4.9^{b}$ & 2.6 & $1.5-4.4^{b}$ \\
\hline Unipolar depression & 2.9 & $1.8-4.7^{b}$ & 2.7 & $1.6-4.5^{\mathrm{b}}$ \\
\hline Any paternal psychiatric illness & 2.4 & $1.6-3.5^{\mathrm{b}}$ & 1.9 & $1.2-2.9^{\mathrm{b}}$ \\
\hline Labour/delivery complications & 1.5 & $1.0-2.1^{b}$ & 1.4 & $1.0-2.0$ \\
\hline Pregnancy complications & 1.2 & $0.8-1.7$ & - & - \\
\hline
\end{tabular}


Table 4 Intellectual disability of likely biomedical basis ${ }^{a}$ (rate per 10000 births, $n=6303$ )

\begin{tabular}{|c|c|c|c|c|c|c|c|c|}
\hline & \multicolumn{8}{|c|}{ Maternal psychiatric status } \\
\hline & \multicolumn{2}{|c|}{ Schizophrenia } & \multicolumn{2}{|c|}{ Bipolar disorder } & \multicolumn{2}{|c|}{ Unipolar depression } & \multicolumn{2}{|c|}{ Non-psychiatric comparison } \\
\hline & $n$ & rate & $n$ & rate & $n$ & rate & $n$ & rate \\
\hline X-linked & 0 & - & 2 & 15.4 & 2 & 15.9 & 1 & 3.2 \\
\hline Other chromosomal & 2 & 32.4 & 6 & 46.1 & 5 & 39.8 & 4 & 12.8 \\
\hline Teratogenic & 0 & - & 1 & 7.7 & 0 & - & 0 & - \\
\hline Central nervous system defect & 1 & 16.2 & 0 & - & 0 & - & 1 & 3.2 \\
\hline $\begin{array}{l}\text { Total children with intellectual disability } \\
\text { of likely biomedical basis }\end{array}$ & 3 & 48.5 & 9 & 69.2 & 7 & 55.8 & 6 & 19.2 \\
\hline Total children & 618 & - & 1301 & & 1255 & - & 3129 & - \\
\hline
\end{tabular}

hospital for convulsions was again only significant in the maternal unipolar depression group.

\section{Discussion}

\section{Main findings}

These data add an epidemiological perspective to an accumulating body of evidence of an association between pervasive cognitive deficits and schizophrenia ${ }^{25,26}$ and support findings from a number of studies of an excess of multiply-affected families. Moreover, they supplement recent data showing covariation between a number of neuropsychiatric disorders including schizophrenia, intellectual disability, autism and epilepsy. Our data do not include psychosis, a potential long-term outcome for these children. However, the determination of caseness was based on maternal psychosis, examined separately for maternal schizophrenia, bipolar disorder and unipolar major depression, thus permitting an examination of both the role of familiality in the outcomes investigated and the specificity across the three maternal diagnostic groups.

Several findings stand out. First, children of mothers with schizophrenia, bipolar disorder and unipolar major depression were up to three times more likely to have intellectual disability relative to comparison children of mothers with no psychiatric history.

Second, multivariate analysis suggests that parental history of intellectual disability, maternal severe mental illness (whether it be schizophrenia, bipolar disorder or unipolar major depression), paternal history of psychiatric illness and some obstetric complications may be independent risk factors for intellectual disability in offspring. Although labour and delivery complications just failed to reach significance, the elevated odds ratio of 1.4 (95\% CI 1.0-2.0) dropped little from a significant odds ratio of 1.5 (95\% CI 1.0-2.1) in the univariate analysis. Moreover, individual obstetric complications, neonatal encephalopathy $(\mathrm{OR}=7.7,95 \%$ CI 3.0-20.2) and fetal distress (OR $=1.8,95 \%$ CI 1.1-2.7) were significant predictors of intellectual disability in offspring. The independence of familial and environmental risks in these data is supported by findings from autism research that suggest that prenatal environmental risk factors and parental psychopathology act independently on the risk for autism. ${ }^{27}$

Third, a number of outcomes were differentially distributed depending on maternal diagnosis. Across the full sample, rates of intellectual disability of likely biomedical origin (genetic, metabolic, teratogenic) were higher in case children than

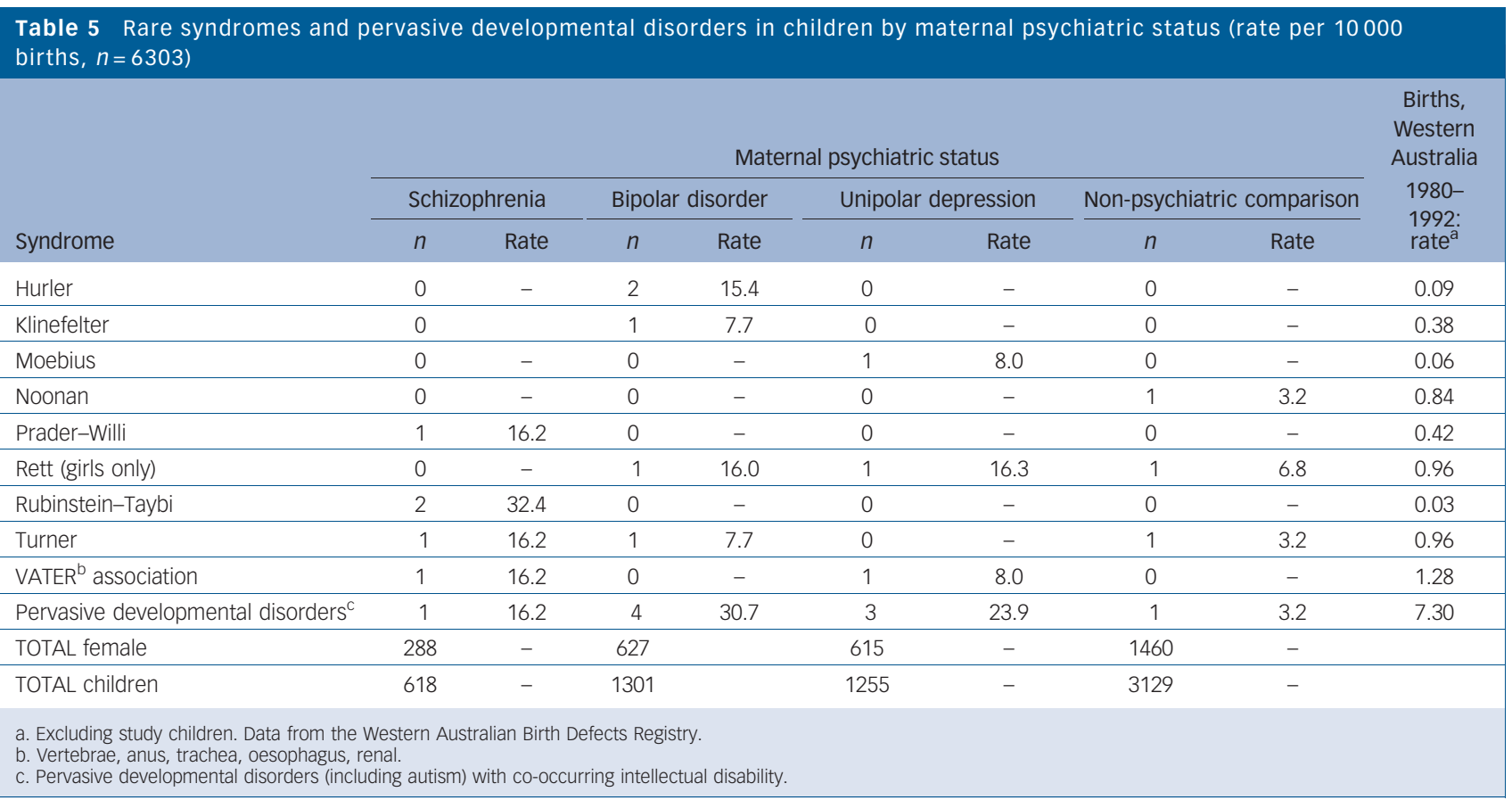


comparison children. Moreover, all groups of case children were affected by rare syndromes at rates well above population rates, although the number affected was small. However, individual syndromes clustered within different groups. Of note, the risk of pervasive developmental disorders was increased tenfold in the maternal bipolar disorder group (significant). Large odds ratios for children of mothers with schizophrenia and unipolar major depression were not significant, with very wide confidence intervals straddling both sides of unity. Rates of epilepsy were elevated in case children whose mothers had been diagnosed with affective psychoses, significantly in those in the maternal unipolar depression group. Of interest in this context, there are several lines of evidence for an association between epilepsy and schizophrenia, ${ }^{28}$ with weaker evidence for the association between epilepsy and bipolar disorder. ${ }^{29}$ We found no association between maternal schizophrenia and epilepsy in children. Although our current data are limited to the risk of epilepsy in offspring of mothers with schizophrenia, future analyses of a larger data-set under construction will enable us to assess not only the relationship between epilepsy and psychosis in the children themselves, but also its specificity to schizophrenia as well as the role of parental history of psychosis and/or epilepsy.

Finally, the importance of the timing of maternal illness onset relative to the index birth varied. The risk of intellectual disability for the children of mothers with schizophrenia and unipolar major depression remained the same irrespective of whether their mothers were first diagnosed with a psychotic illness before or after the index birth. However, for children of mothers with bipolar disorder, the risk of intellectual disability was significantly increased only if onset of maternal illness pre-dated the index birth. This important finding may reflect the role of medication taken during pregnancy. For example, sodium valproate prescribed as a mood stabiliser in bipolar disorder has been associated with an increased risk of congenital anomalies in the foetus when used in pregnancy. ${ }^{30}$ In the absence of specific evidence, this finding is indicative only, and further investigation is warranted.

These findings add to a body of literature (see online Table DS4) reporting associations between a number of syndromes and psychotic disorders. This includes well-established findings of congenital anomalies, psychiatric and behavioural phenotypes associated with a microdeletion on chromosome 22q11.2 in velocardiofacial syndrome. ${ }^{31}$ It was not possible to identify velocardiofacial syndrome in the children in our study as the recording of this syndrome on registers in the time period 1980-1992 was poor. However, we have previously reported data on the same cohort showing that children of mothers with schizophrenia were more likely than comparison children to experience cardiovascular birth defects, including a threefold increase in ventricular septal defect, which is characteristic of velocardiofacial syndrome. ${ }^{12}$ The same effect was not found for the children in the maternal bipolar disorder or maternal unipolar depression groups. We have also previously reported that children of women with schizophrenia, but not other case children, have a higher rate of minor physical anomalies than children of unaffected mothers. Such abnormalities are indicative of neurodevelopmental disruption and are found more frequently in people with schizophrenia. ${ }^{32}$ The specificity of both the cardiac abnormalities and the minor physical anomalies to the schizophrenia group, as well as the fact that they were significantly elevated irrespective of the timing of onset of maternal psychosis, are suggestive of a genetic vulnerability.

\section{Genetic and environmental risk factors}

How do these data fit with recent reports on the partially shared genetics of neuropsychiatric disorders, and what explanatory mechanisms best account for our findings? Researchers probing the genetics of complex diseases are providing new insights into the individual and familial clustering of disorders such as schizophrenia and intellectual disability that has been observed by epidemiologists. In a recent analysis of comorbidity among 161 medical conditions using data from 1.5 million patient records, Rzhetsky et al ${ }^{33}$ detected a highly connected network of strong pair-wise correlations indicating that schizophrenia, bipolar disorder, epilepsy and autism may share genetic overlaps. Genome-wide association studies have been important in identifying common polymorphisms affecting disease susceptibility. Recent studies of genomic structural variation report an excess copy number variant load in schizophrenia, but not bipolar disorder, compared with controls, with specific rare copy number variations conferring risk for multiple neuropsychiatric outcomes, including schizophrenia. ${ }^{34}$ Deletions at $15 \mathrm{q} 13.3$, for example, robustly associated with schizophrenia, ${ }^{35}$ are also associated with autism and epilepsy. ${ }^{34}$ Some of the inherited and de novo mutations may be highly penetrant. Although their specific role in the pathogenesis of the associated disorders remains unclear, many of the disrupted genes are along shared neurodevelopmental pathways. ${ }^{4,5}$ This suggests a common pathogenic mechanism acting in utero or early infancy.

Obstetric complications have long been implicated in the aetiology of schizophrenia ${ }^{36}$ and continue to be among the most consistently reported environmental risk factors for schizophrenia. ${ }^{37}$ As risk factors, they sit comfortably within a neurodevelopmental framework wherein pre- or perinatal disruption of normal central nervous system development manifests itself in adulthood as schizophrenia, but also gives rise to other deficits in childhood and early adolescence. Indeed, obstetric complications play an important role in non-syndromal intellectual disability, ${ }^{38}$ autism ${ }^{39}$ and epilepsy. ${ }^{40}$ Environmentally induced changes in the uterine environment such as increased maternal cytokines in response to hypoxia ${ }^{41}$ or infection, ${ }^{42}$ or stress-induced elevation of circulating corticosteroid levels ${ }^{43}$ may contribute to the risk of adverse neuropsychiatric outcomes in offspring through their potential to disrupt neuronal development. Yet little is known about how these environmental insults, multiple susceptibility genes and stochastic epigenetic events interact. It is possible that environmental risk factors such as hypoxia-ischaemia, infection and fetal malnutrition modify the penetrance and phenotypic expression of potentially pathogenic mutations in utero. Nicodemus et al found that significant interactions between serious obstetric complications and polymorphisms in genes regulating hypoxia-ischaemia influenced the risk for schizophrenia in a family-based study of transmission disequilibrium. ${ }^{44}$ Meanwhile, animal studies indicate that timing is critical to the specific outcome. ${ }^{45}$ Our ecologic study of in utero exposure to influenza and subsequent risk of schizophrenia, affective psychosis and intellectual disability also provides limited support for the differential effect of trimester of exposure on outcome. ${ }^{46}$ One hypothesis is that obstetric events act additively or multiplicatively with genetic factors to disrupt central nervous system development; the variation in outcomes across children may reflect different genetic risk and differences in the nature and timing of the in utero exposure. Our data indicate both a degree of commonality across maternal high-risk groups as well as some specificity, depending on the outcome of interest. Moreover, in our multivariate model of the risk of intellectual disability, familial and environmental factors appear to act independently. We note, however, that family history variables are only a proxy for genetic status and that multivariate analyses using family history variables cannot definitively establish the independence of genetic and environmental risk factors. 


\section{Strengths and limitations}

This study makes an important contribution to other studies of high-risk children of women with schizophrenia and other psychoses by virtue of its capacity to: ascertain intellectual disability and other neuropsychiatric outcomes in these children; assess the impact of obstetric risk factors on outcomes; determine familial liability for intellectual disability as well as psychosis; and examine specificity to schizophrenia. As a population-based register study, it has the advantage of being able to access prospectively collected longitudinal data for a large number of children on a variety of predictors and outcomes. One limitation is the absence of data on antipsychotic medication use in pregnancy. The size and lipophilicity of these molecules increases the likelihood that they will cross the placenta ${ }^{47}$ and, as discussed earlier, there are indications that some of these medications increase the risk of congenital anomalies and developmental delay. Another limitation is the reliance on the psychiatric case register to determine a family history of psychosis. Although the register is more comprehensive than many others worldwide, covering in-patient, out-patient and ambulatory care contacts, it does not include contacts with general practitioners or with private psychiatrists and psychologists outside of the hospital setting. It is possible that some comparison children were misclassified and, in fact, had an affected parent with psychosis. To minimise misclassification, especially with respect to unipolar depression, we have only included more severe cases by restricting our coverage to diagnoses in the ICD-9 296.x range for affective disorders and not including 300.4 (neurotic depression) and 311 (depressive disorder not elsewhere classified). However, the impact of the misclassification would be conservative, attenuating differences between comparison and case children.

A further limitation is possible misclassification of paternity. The median rate of paternal misclassification in cases not selected on the basis of disputed paternity has been estimated at $3.7 \%{ }^{48}$ There is no reason to expect that record linkage studies are more prone to errors with respect to biological paternity than any other form of data gathering relying on self-reported information. However, a different problem arises if no father is named on the birth registration certificate. This affected $7.0 \%$ of children on our database. Birth records that had no father registered were differentially distributed and were more likely to occur in birth registrations for case children, especially the children of women with schizophrenia (20.9\% compared with $8.5 \%, 5.1 \%$ and $4.3 \%$ respectively for maternal bipolar disorder, maternal unipolar disorder and the comparison children). Potentially, this could lead to underreporting of paternal psychiatric histories if assortative mating is common in women with psychotic disorders. Such a bias would attenuate the strength of any association.

Finally, despite the cohort size, small numbers of cases for some outcomes resulted in wide confidence intervals around the estimates. We are currently in the process of compiling a dataset of almost half a million children born between 1980 and 2001. This much expanded cohort will increase our statistical power substantially. Moreover, in the future, we will be able to use this enlarged cohort to investigate distal outcomes in the children such as schizophrenia and other psychotic disorders and look at their relationship to neuropsychiatric outcomes more proximal to the intra-uterine and neonatal periods, including birth defects, rare syndromes, intellectual disability and pervasive developmental disorders. ${ }^{13}$

In conclusion, our data support growing evidence that phenotypically different neuropsychiatric disorders cluster within pedigrees and that this is likely to result, in part, from some genetic overlap between disorders and, in part, from exposure to certain types of obstetric trauma and other environmental insults. However, much remains to be learned about the role of genetic, epigenetic and environmental risk factors, and their interaction, in the range of neuropsychiatric outcomes.

\section{Funding}

This work was supported by the National Health and Medical Research Council (303235, 458702); March of Dimes (12-FY04-48, 12-FY07-224); and Stanley Medical Research Institute (00-253).

\section{Acknowledgements}

We thank Tom Pinder (Western Australian Department of Health) who supplied psychiatric case register data and provided guidance on their use and interpretation, and Helen Leonard (Telethon Institute for Child Health Research) who validated register data on intellectual disability level and Heber diagnosis against case records.

Vera A. Morgan, BA, MSOcSC, PhD, School of Psychiatry and Clinical Neurosciences, and Centre for Clinical Research in Neuropsychiatry, The University of Western Australia; Maxine L. Croft, PhD, BAppSc, School of Psychiatry and Clinical Neurosciences, and Telethon Institute for Child Health Research, Centre for Child Health Research, The University of Western Australia; Giulietta M. Valuri, Grad Dip Epi, BBus, INP, School of Psychiatry and Clinical Neurosciences, The University of Western Australia; Stephen R. Zubrick, MSC, MA, PhD, Telethon Institute for Child Health Research, Centre for Child Health Research, The University of Western Australia; Carol Bower, MBBS, MSC, PhD, FAFPHM, DLSHTM, FPHAA, Telethon Institute for Child Health Research, Centre for Child Health Research, The University of Western Australia, and Western Australian Register of Developmental Anomalies, King Edward Memorial Hospital, Australia; Thomas F. McNeil, PhD, School of Psychiatry and Clinical Neurosciences, The University of Western Australia, Australia, and Skånes University Hospital, Lund, Sweden; Assen V. Jablensky, MD, DMSC, FRANZCP, FRCPsych, Centre for Clinical Research in Neuropsychiatry, and School of Psychiatry
Fiver and Clinical Neurosciences, The University of Western Australia, Australia

Correspondence: Professor Vera A. Morgan, School of Psychiatry and Clinical Neurosciences, The University of Western Australia, Level 3 Medical Research Foundation Building, Rear 50 Murray Street, Perth, 6000, Western Australia, Australia. Email: Vera.Morgan@uwa.edu.au

First received 29 Mar 2011, final revision 6 Oct 2011, accepted 26 Oct 2011

\section{References}

1 Xu B, Roos JL, Levy S, van Rensburg EJ, Gogos JA, Karayiorgou M. Strong association of de novo copy number mutations with sporadic schizophrenia. Nat Genet 2008; 40: 880-5.

2 Geschwind DH, Konopka G. Neuroscience in the era of functional genomics and systems biology. Nature 2009; 461: 908-15.

3 Crespi B, Stead P, Elliot M. Comparative genomics of autism and schizophrenia. Proc Natl Acad Sci USA 2010; 107 (suppl 1): 1736-41.

4 Guilmatre A, Dubourg C, Mosca A-L, Legallic S, Goldenberg A, Drouin-Garraud $V$, et al. Recurrent rearrangements in synaptic and neurodevelopmental genes and shared biologic pathways in schizophrenia, autism, and mental retardation. Arch Gen Psychiatry 2009; 66: 947-56.

5 Walsh T, McClellan JM, McCarthy SE, Addington AM, Pierce SB, Cooper GM, et al. Rare structural variants disrupt multiple genes in neurodevelopmental pathways in schizophrenia. Science 2008; 320: 539-43.

6 Kraepelin E. Dementia Praecox and Paraphrenia. R. E. Krieger Publishers, 1919.

7 Penrose LS. A Clinical and Genetic Study of 1280 Cases of Mental Defect. Medical Research Council: Special Report Number 229. HMSO, 1938.

8 Hemmings CP. Schizophrenia spectrum disorders in people with intellectual disabilities. Curr Opin Psychiatry 2006; 19: 470-4.

9 Morgan VA, Leonard H, Bourke J, Jablensky AV. Intellectual disability co-occurring with schizophrenia and other psychiatric illness: populationbased study. Br J Psychiatry 2008; 193: 364-72.

10 Harris J. Intellectual Disability. Understanding its Development, Causes, Classification, Evaluation, and Treatment. Oxford University Press, 2006.

11 Jablensky A. The epidemiological horizon. In Schizophrenia (2nd edn) (eds SR Hirsch, DR Weinberger): 203-31. Blackwell, 2003.

12 Jablensky A, Morgan VA, Zubrick S, Bower C, Yellachich L. Pregnancy, delivery, and neonatal complications in a population cohort of women with schizophrenia and major affective disorders. Am J Psychiatry 2005; 162: 79-91. 
13 Morgan VA, Valuri GM, Croft ML, Griffith JA, Shah S, Young DJ, et al. Cohor profile: pathways of risk from conception to disease: the Western Australian schizophrenia high-risk e-Cohort. Int J Epidemiol 2010; November 19: Epub ahead of print.

14 Glasson EJ, de Klerk NH, Bass AJ, Rosman DL, Palmer LJ, Holman CDJ. Cohort profile: The Western Australian Family Connections Genealogical Project. Int J Epidemiol 2008; 37: 30-5.

15 Croft ML, Read AW, de Klerk N, Hansen J, Kurinczuk JJ. Population based ascertainment of twins and their siblings, born in Western Australia 1980 to 1992, through the construction and validation of a maternally linked database of siblings. Twin Res 2002; 5: 317-23.

16 World Health Organization. International Statistical Classification of Diseases and Related Health Problems, Ninth Revision (ICD-9). WHO, 1977.

17 Castle D, Jablensky A, McGrath J, Carr V, Morgan V, Waterreus A, et al. The Diagnostic Interview for Psychoses (DIP): development, reliability and applications. Psychol Med 2006; 36: 69-80.

18 Heber R. A manual on terminology and classification in mental retardation. Am J Ment Defic 1959; 64 (suppl): 1-111.

19 Yeargin-Allsopp M, Murphy C, Cordero J, Decouflé P, Hollowell J. Reported biomedical causes and associated medical conditions for mental retardation among 10-year-old children, metropolitan Atlanta, 1985 to 1987. Dev Med Child Neurol 1997; 39: 142-9.

20 Badawi N, Kurinczuk JJ, Keogh JM, Alessandri LM, O'Sullivan F, Burton PR, et al. Antepartum risk factors for newborn encephalopathy: the Western Australian case-control study. BMJ 1998; 317: 1549-53.

21 McNeil TF, Sjöström K. McNeil-Sjöström Scale for Obstetric Complications. Lund University, 1995.

22 World Health Organization. The ICD-10 Classification of Mental and Behavioural Disorders: Clinical Descriptions and Diagnostic Guidelines. WHO 1992

23 Malaspina D, Harlap S, Fennig S, Heiman D, Nahon D, Feldman D, et al. Advancing paternal age and the risk of schizophrenia. Arch Gen Psychiatry 2001; 58: 361-7.

24 Reichenberg $\mathrm{A}$, Gross $\mathrm{R}$, Weiser $\mathrm{M}$, Bresnahan $\mathrm{M}$, Silverman J, Harlap $\mathrm{S}$, et al. Advancing paternal age and autism. Arch Gen Psychiatry 2006; 63: 1026-32.

25 Heinrichs RW, Zakzanis KK. Neurocognitive deficit in schizophrenia: a quantitative review of the evidence. Neuropsychology 1998; 12: 426-45.

26 Hallmayer JF, Kalaydjieva L, Badcock J, Dragovic M, Howell S, Michie PT, et al Genetic evidence for a distinct subtype of schizophrenia characterized by pervasive cognitive deficit. Am J Hum Genet 2005; 77: 468-76.

27 Larsson HJ, Eaton WW, Madsen KM, Vestergaard M, Olesen AV, Agerbo E, et al. Risk factors for autism: perinatal factors, parental psychiatric history, and socioeconomic status. Am J Epidemiol 2005; 161: 916-25.

28 Cascella N, Schretlen D, Sawa A. Schizophrenia and epilepsy: is there a shared susceptibility? Neurosci Res 2009; 63: 227-35.

29 Mula M, Marotta A, Monaco F. Epilepsy and bipolar disorders. Expert Rev Neurother 2010; 10: 13-23.

30 Perucca E, Tomson T. Prenatal exposure to antiepileptic drugs. Lancet 2006 367: 1467-9.
31 Bassett A, Chow E, AbdelMalik P, Gheorghiu M, Husted J, Weksberg R. The schizophrenia phenotype in 22q11 Deletion Syndrome. Am J Psychiatry 2003; 160: $1580-6$.

32 Ismail B, Cantor-Graae E, McNeil T. Minor physical anomalies in schizophrenia patients and their siblings. Am J Psychiatry 1998; 155 1695-702.

33 Rzhetsky A, Wajngurt D, Park N, Zheng T. Probing genetic overlap among complex human phenotypes. PNAS 2007; 104: 11694-9.

34 Grozeva D, Kirov G, Ivanov D, Jones IR, Jones L, Green EK, et al. Rare copy number variants: a point of rarity in genetic risk for bipolar disorder and schizophrenia. Arch Gen Psychiatry 2010; 67: 318-27.

35 Stefansson H, Rujescu D, Cichon S, Pietiläinen OP, Ingason A, Steinberg S, et al. Large recurrent microdeletions associated with schizophrenia. Nature 2008; 455: 232-6.

36 Rosannoff A, Handy L, Rosanoff I, Brush S. The etiology of so-called schizophrenic psychoses: with special reference to their occurrence in twins. Am J Psychiatry 1934; Sept: 247-86.

37 Cannon M, Jones PB, Murray RM. Obstetric complications and schizophrenia: historical and meta-analytic review. Am J Psychiatry 2002; 159: 1080-92.

38 Leonard $\mathrm{H}$, Wen $\mathrm{X}$. The epidemiology of mental retardation: challenges and opportunities in the new millennium. Ment Retard Dev Disabil Res Rev 2002; 8: 117-34.

39 Hultman $\mathrm{CM}$, Sparen $\mathrm{P}$, Cnattingius S. Perinatal risk factors for infantile autism. Epidemiology 2002; 13: 417-23.

40 Scher M. Prenatal contributions to epilepsy: lessons from the bedside. Epileptic Disord 2003; 5: 77-91.

41 Tsukimori K, Nakano $\mathrm{H}$, Wake $\mathrm{N}$. Difference in neutrophil superoxide generation during pregnancy between preeclampsia and essential hypertension. Hypertens Res 2007; 49: 1436-41.

42 Brown AS. Prenatal infection as a risk factor for schizophrenia. Schizophr Bull 2006; 32: 200-2.

43 Goodyer IM, Park RJ, Netherton CM, Herbert J. Possible role of cortisol and dehydroepiandrosterone in human development and psychopathology. Br J Psychiatry 2001; 179: 243-9.

44 Nicodemus KK, Marenco S, Batten AJ, Vakkalanka R, Egan MF, Straub RE, et al. Serious obstetric complications interact with hypoxia-regulated/ vascular-expression genes to influence schizophrenia risk. Mol Psychiatry 2008; 13: 873-7.

45 Meyer U, Yee BK, Feldon J. The neurodevelopmental impact of prenatal infections at different times of pregnancy: the earlier the worse? Neuroscientist 2007; 13: 241-56.

46 Morgan VA, Castle D, Page A, Fazio S, Gurrin L, Burton P, et al. Influenza epidemics and the incidence of schizophrenia, affective disorders and mental retardation in Western Australia: no evidence of a major effect. Schizophr Res 1997; 26: 25-39.

47 Pinkofsky HB. Psychosis during pregnancy: treatment considerations. Ann Clin Psychiatry 1995; 9: 175-9.

48 Bellis MA, Hughes K, Hughes S, Ashton JR. Measuring paternal discrepancy and its public health consequences. J Epidemiol Community Health 2005; 59 749-54. 